\title{
La desmovilización como parámetro para la implementación de la teoría de la igualdad en el proceso de reintegración*
}

\author{
Demobilization as a measurement to implement equality \\ theory in reintegration processes
}

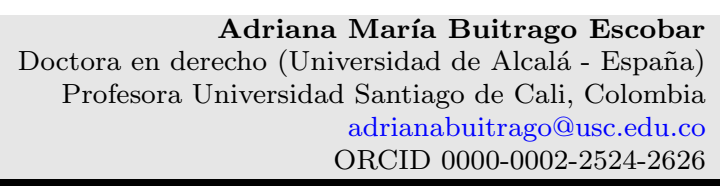

Recibido: 22/11/17 Aprobado 06/03/18

DOI: http://dx.doi.org/10.25054/16576799.1872

\section{RESUMEN}

Con la desmovilización del grupo armado FARC-EP (Fuerzas Armadas Revolucionarias de Colombia - Ejército del Pueblo), tras la firma del proceso de paz, se debe estar preparados para el futuro y evitar incurrir en los errores del pasado. La última desmovilización masiva fue en el proceso de paz con los paramilitares (Autodefensas Unidas de Colombia, en adelante AUC) en el 2005, donde los indicadores, no fueron muy alentadores: De casi cincuenta mil desmovilizados de las AUC, solo nueve mil de ellos culminaron procesos, y otros siete mil a esta fecha, aún se encuentran estancados en el proceso; porcentaje que no alcanza a ser siquiera la mitad de la población en cuestión ${ }^{1}$.

Este fenómeno incide negativamente en la finalidad misma de los procesos: al no tener cifras exitosas de reintegración, no existen garantías de acceso al trabajo para los desmovilizados, que debido a su condición de ex militantes, se enfrentan a varios obstáculos en el mundo laboral. El sector privado suele ser sumamente discriminante al momento de vincular reintegrados a puestos donde deben tratar directamente con el cliente o como representación de la empresa. Esto da como resultado, una posibilidad limitada de vinculación laboral, lo que suele desencadenar el regreso a la subversión.

\section{PALABRAS CLAVE}

AUC; Desmovilización; Grupos Armados; Igualdad; Proceso de Paz.

\section{ABSTRACT}

With the demobilization of the armed group FARC-EP (by its Spanish acronym) and the signing of the peace treaty, we must prepare ourselves for the future and avoid repeating the mistakes from the past. The last massive demobilization was back in 2005 in the signing of the peace treaty with the Paramilitaries (Autodefensas Unidas de Colombia, from here on AUC) where the numbers weren't encouraging; from almost fifty thousand demobilized AUC, only nine thousand finished the process, and seven thousand to this date are still stuck in the process; percentage which is not even half of the population in question.

This phenomenon affects negatively the reason of the peace treaties: since there is no successful amount of reintegration processes, there can be no guaranties to access jobs for the demobilized. That due to the fact of their past as ex combatants, they are forced to face serious obstacles in the labor world. The private sector tends to be very discriminative at the moment of hiring reintegrated people in jobs where they have direct interaction with the client or the representation of the company. This results in a limited possibility of landing a job, what gives as a final result in the regression to subversion.

*Artículo de Investigación

Proyecto finalizado. Universidad Santiago de Cali. Adriana Maria Buitrago Escobar (Autora) y Michelle Ariana Ospina Giraldo (Co-autora). Grupo de investigación GICPODERI, semillero RELADEFUN.

${ }^{1}$ Cifras Agencia de Reintegración y Normalización, 2016. 


\section{KEYWORDS}

AUC; Armed Groups; Demobilization; Equality; Peace Process.

\section{INTRODUCCIÓN}

En diversos países alrededor del mundo se han llegado a implementar procesos de desmovilización al finiquitar un conflicto interno. Todos con la esperanza de forjar el camino hacia una mejor sociedad, aunque la mayoría fallidos dado las exigencias de conversión social y cultural que requieren, con el ingrediente adicional que la mayoría de países con este tipo de situaciones son países en vía de desarrollo, con diversos problemas económicos, sociales y legislativos. Al momento en que se inicia un proceso de desmovilización, una población de ciudadanos que antes no se incluía en los diversos procesos sociales de un país, siente el brusco cambio que implica volver a la legalidad.

Sin embargo, el cambio puede ser más inclusivo si se adoptan políticas y procesos diseñados integralmente para dicha población desmovilizada, garantizándole así una oportunidad de reingreso. Es en este momento en donde la implementación de principios o teorías, como la de la igualdad, entran a jugar un papel decisivo en el proceso; una igualdad en diversas áreas como por ejemplo la laboral, y se podría llegar a decir que debe ser una igualdad discriminativa en el momento en el que, no se puede evaluar a los sujetos desmovilizados con los mismos criterios con que se evalúa un ciudadano común.

De esta manera, los obstáculos que atentan contra un proceso éxitos de reintegración implican:

[... la reinserción en la vida civil normal de un elevado número de grupos de población (combatientes desmovilizados, poblaciones internamente desplazadas, refugiados y la población residente afectada por el conflicto). Ello implica también la reactivación de la economía afectada por el conflicto, la creación de nuevas bases para obtener fuentes seguras de ingresos y la reconstitución de las instituciones sociales y políticas (Organización Internacional del Trabajo, 2002, p.5).

Observando el proceso de reintegración que se llevó acabo con los desmovilizados de las AUC, se observa que la falta de aplicación de la teoría de la igualdad, pudo haber sido un factor decisivo en el fracaso de este proceso. Reintegrar a una persona al margen de la ley nuevamente a la dinámica social de un país desigual termina siendo más problemático de lo pensado.

Desde agosto de 2002 y hasta enero de 2010 se han desmovilizado 52.403 personas,
31.671 procedentes de grupos paramilitares que dejaron las armas de manera colectiva, y 20.732 miembros de grupos guerrilleros y paramilitares que se desmovilizaron individualmente. Según información de la Policía Nacional, entre el año 2001 y el mes de diciembre de 2009 han sido capturados 5.890 desmovilizados y han muerto 2.210 (Barragán. L., A., Universidad Militar Nueva Granada, 2012).

El gobierno tiene agencias y procesos encaminados para llevar a cabo esta tarea; sin embargo, no tiene los postulados ni las normativas suficientes para hacer de este proceso efectivo en su totalidad. No basta con que el desmovilizado se reintegre a la sociedad si la sociedad y el Estado no tienen como asegurarle sus derechos básicos, como a los demás ciudadanos. La legislación solo está pensada en cómo desmovilizarlos, más no verdaderamente en como reintegrarlos.

[...] "en relación constante con ese modelo individualista están los excombatientes de grupos armados paramilitares y guerrilleros. Si bien existen excombatientes que no encuentran dificultades en la interacción con los programas estatales de DDR, si existen otros que por su formación política y su experiencia de vida cuestionan y problematizan la relación puesto que se impide su participación en la definición de la política pública y en la construcción conjunta de la sociedad. Ellos plantean de DDR al centrarse en un modelo individualista niega el carácter de sujetos políticos a los excombatientes (Medina-Arbeláez, C. (2007) obtenido de Institut gouvernance (2016).

Esto solo se puede lograr a través de políticas públicas encaminadas a una igualdad de oportunidades, atención, derechos. Bobbio, N. (1993) sostiene que la igualdad es una condición necesaria para la justicia. Se podría afirmar entonces, como lo sostiene Ribotta, S. (2010) en defensa de Bobbio, que la teoría desarrollada por él se enmarca en una concepción del Estado Social de Derecho, Estado de concepción liberal, pregonero de derechos y deberes, cimentado sobre postulados como la dignidad humana, la integridad y la prevalencia del interés general (Ribotta, S., 2010).

\section{CONTEXTO}

En el caso puntual de la reintegración de los desmovilizados, por tratarse de un asunto que requiere de la convergencia de todos los actores sociales y al procurar atender los aspectos económicos, sociales y 
comunitarios de los desmovilizados que optan por estos beneficios, amerita un esfuerzo que involucra a toda la sociedad y en especial al sector empresarial (Rettberg, A., 2012), principalmente en la creación de oportunidades laborales que garanticen su acceso al empleo y a fuentes seguras de empleo. Esto teniendo en cuenta que es precisamente allí, en la atención de aspectos como el económico, que logran identificarse barreras que limitan entre otras cosas, el pleno ejercicio del derecho al trabajo para esta población y la emergencia de nuevos desafíos para la competitividad de las empresas a la luz de un contexto social que implica adaptar sus diseños estratégicos.

Tal aseveración parte de considerar escenarios como el colombiano donde según cifras de la Agencia para la Reintegración y Normalización, hay alrededor de 22.000 personas que se encuentran en ese proceso, de las cuales 4.900 están ocupados en el sector formal, 10.559 en el sector informal, aproximadamente 10.000 constituyen población económicamente inactiva, y cerca de 3.900 están desocupados (Agencia Colombiana para la Reintegración, 2015). Es decir, que alrededor de 14.536 personas en proceso de reintegración no cuentan con opciones económicas.

Sumado a lo anterior, la situación laboral de los excombatientes sigue presentando falencias que tienen que ver con las dificultades para ubicarse en trabajos formales, Con la ausencia de políticas de empleo enfocadas a esta población en particular, con el ofrecimiento de otras opciones de ingresos por parte de grupos ilegales emergentes (OIT, 2002). Esto último dado que "las ofertas económicas por parte de las organizaciones armadas ilegales, por ejemplo, sumadas a las dificultades para emplearse en la legalidad, configuran una de las causales por las cuales una persona en proceso de reintegración decide reincidir en acciones delictivas" (García, A. (2015.) p.2). Así también, la percepción del sector empresarial sobre esta población, constituye una serie de limitantes del derecho al trabajo, donde según lo mencionado por Roldán, L. (2013) en los resultados de la investigación adelantada sobre este tema:

[...] los sectores que no tienen tendencia a vincular dentro de su nómina personas desmovilizadas argumentan principalmente que su condición de desmovilizados no es compatible con la naturaleza del servicio prestado, inseguridad de sus clientes y de la empresa, que no son un personal profesionalmente calificado, la confidencialidad de la información que manejan, la falta de confianza y el miedo e intranquilidad que les generan, los antecedentes de estas personas y porque prefieren elegir entre otras opciones (p.115).

Tales hallazgos, dan cuenta de una serie de obstáculos que los excombatientes encuentran en su proceso de reintegración social y vinculación laboral; que si bien están representadas en políticas de selección de personal y lineamientos internos de las empresas, redundan en situaciones discriminatorias de las relaciones laborales. Lo anterior debido a que la discriminación en estas relaciones involucra "cualquier distinción, exclusión o preferencia que produzca el rechazo o la desigualdad en las oportunidades o en el trato de solicitudes de empleo o de ocupación realizada por razón de raza, color, sexo, religión, opiniones políticas, nacionalidad de origen o extracción social" (Red Pacto Mundial España, 2015).

Aterrizando en el modelo colombiano, un estudio del 2013, sobre el proceso de reintegración de desmovilizados, podemos rescatar lo siguiente:

[...] "De los más de 35.000 desmovilizados, apenas 1.733 han logrado vincularse laboralmente gracias a acciones de la Agencia Colombiana de Reintegración. Esta carencia de oportunidades de empleo y la poca protección laboral a los desmovilizados han conducido a que se incrementen los niveles de violencia en los distintos centros urbanos y también al rearme de estas personas, dando lugar al surgimiento de fenómenos como las BACRIM" (Roldan, L. 2013, p. 109).

\section{EJEMPLO HISTORÍCO: DESMOVILIZACIÓN DE LAS AUC}

LA

En el 2002, a la par en que Álvaro Uribe Vélez asumía la presidencia del país, se inició la implementación de políticas de desarme, desmovilización y reintegración (sus siglas DDR). Era un proceso abierto, optativo, para guerrilleros y combatientes de los distintos grupos armados que existan en el país.

Para Naciones Unidas, el DDR es un proceso que contribuye a la seguridad y estabilidad en contextos de recuperación posbélica a través de la eliminación de las armas a manos de los combatientes de las estructuras militares y ayudándoles a reintegrarse social y económicamente en la sociedad, buscándoles modos de vida civiles. Para este organismo, el DDR es parte de la aproximación multidimensional de la construcción de la paz postconflicto y de la reconstrucción, y puede contribuir a construir seguridad. [..] El DDR, por tanto, por sí sólo, no comporta la paz, sino que la facilita. Es un elemento más de un proceso más amplio con muchos compromisos, aunque el más importante de ellos es preparar a los excombatientes para que sean instrumentos de paz (Fisas, V., 2011). 
Posteriormente, en el 2003, Colombia logra terminar uno de sus latentes conflictos con uno de los más sanguinarios grupos ilegales: Las autodefensas unidas de Colombia (AUC). Con el acuerdo de Santa fe de Ralito, firmado el 15 de julio de ese año, se pretendía desmovilizar al grupo paramilitar y a la vez frenar las masacres indiscriminadas que se venían registrando desde el 2002.

Junto con las desmovilizaciones, se crearon entidades gubernamentales, las cuales estarían encargadas de no solo reintegrar al desmovilizado, sino seguir haciendo campañas de desmovilización, reportar cifras de desmovilizados y administrar los fondos otorgados como auxilios económicos para dicha población. Hoy en día, la conocemos como Agencia de Reintegración y Normalización (ARN), sin embargo, comenzó como un programa adscrito al ministerio de Interior y Justicia; el programa de Reincorporación de la vida civil (PRVC), entre los años del 2003 y el 2006.

El PRVC era un programa de corta duración, que tenía como finalidad realizar un proceso parecido al de la ARN hoy en día: reformar, preparar, acompañar y capacitar a los desmovilizados para reintegrarse plenamente en la vida civil. Sin embargo, al crecerse el número de desmovilizados debido al acuerdo firmado entre partes, el PRVC comenzó a tener problemas de congestión, administrativos y operativos, que para poder cumplir con las exigencias de las desmovilizaciones masivas de las AUC hacía necesario una reforma urgente: así evolucionó en septiembre del 2006 la Alta Consejería Presidencial para la reintegración.

Sin embargo, en el puente de esta transición tan importante, no podemos olvidar un gran pilar legislativo, y lo que se convertiría en un punto de referencia, un mapa para este tipo de entidades gubernamentales: la ley 975 del 2005. Las desmovilizaciones eran fenómenos cada vez más frecuentes y en más cantidades, ante esto el legislador vio la necesidad de facilitar los procesos; ese es el alma de la ley, facilitar los procesos de paz y la reincorporación individual y colectiva de miembros de grupos al margen de la ley.

Podríamos afirmar entonces, que durante la existencia de la PRVC y la desmovilización masiva de las AUC, el Estado aceptó y acató la necesidad de volver el programa de reintegración un programa a largo plazo; ampliando sus beneficios y fácil acceso, pero a su vez, haciendo de sus filtros más difícil de pasar, y el proceso con cada vez más duración.

Cinco años después de estas últimas modificaciones, la Alta Consejería pasa a ser en noviembre del 2011 la Agencia Colombiana de Reintegración, una unidad administrativa especial con personería jurídica, con la misma misión; apostarle a la reintegración de las desmovilizaciones por venir. Hoy en día, gracias a la firma de la paz suscrita entre el Gobierno de Santos y las FARC-EP, la ACR volvió a transformarse y hoy día es conocida como la Agencia Reintegración y Normalización (ARN).

Los procesos de la ARN demoran medianamente unos 8 años. Los desmovilizados en cuestión, se reintegran al grupo con más reciente ingreso. Estos procesos pasan por varias etapas que se suponen forman a una persona íntegra: social, económica, psicológica, familiar, educativa, entre otras. La ARN capacita, colabora con auxilios mensuales, apoya en la parte psicosocial y recientemente han comenzado a apoyar con la creación de empresa; si logra cumplir con todos los filtros, tendrá la posibilidad de recibir financiación para sus proyectos.

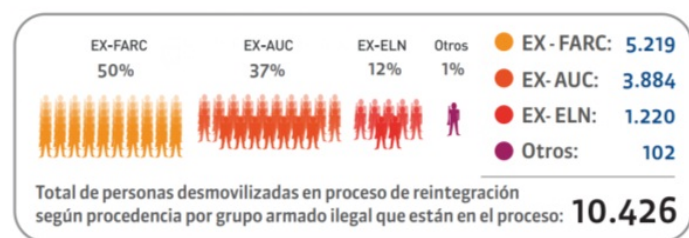

Fuente: Agencia para la reincorporación y la normalización, 2018.

\section{IGUALDAD Y DIGNIDAD HUMANA: EL SUJETO EN CUESTIÓN}

Dentro de los postulados que se debaten en un Estado Social de Derecho, vemos que en estos procesos tan complejos de desmovilizaciones y reintegro, se cuestiona la verdadera igualdad que tienen estas minorías a reintegrarse nuevamente en la vida civil. Como habíamos dicho al comienzo del escrito, Colombia es un país propenso a las desigualdades; desigualdades que la mayoría de la población civil vive día a día, y de las cuales no están exentos estas minorías.

El proceso suena innovador y muy noble así; sin embargo ¿por qué estos indicadores tan desalentadores? Hasta las últimas cifras individuales de la desmovilización de la AUC en el 2016, de casi cincuenta mil hombres que se desmovilizaron, medianamente unos nueve mil completaron su proceso de reintegración. Otros siete mil se encuentran aún en proceso; en aquel limbo de deserción y retorno, y el resto sigue sin reporte o en definitiva reincidieron en la criminalidad, como por ejemplo, uniéndose a las BACRIM.

A marzo de 2018, la ARN registra aproximadamente unos sesenta mil hombres desmovilizados (incluyendo en estos momentos la población de LAS FARC), sin embargo, entre LAS FARC, AUC y ELN, son solo aproximadamente once mil desmovilizados en proceso de reintegración; escasamente un $11 \%$. 
Ahora bien, se considera importante anotar en este momento la diferencia entre el desmovilizado y el reintegrado. La condición del desmovilizado es mucho más incierta que la del reintegrado; cumple con la dejación de armas y la cesación de acciones bélicas, más sin embargo no logra hacer, en la mayoría de casos una adecuada transición hacia la vida civil, encontrándose con problemas de normalización en la sociedad, vinculación laboral, y discriminación, siéndole así más atractivas las ofertas ostentosas de grupos ilegales al margen a la ley; factores decisivos al momento de tomar la decisión de abandonar cualquier tipo de reintegración.

[... Algunos DDR han fracasado y ha sido motivo de nuevas hostilidades, y ello por haber partido de un concepto falso, a saber, pensar que las siglas significaban Derrota, Desprecio y Rendición. Sin embargo, para tener éxito, el DDR en ningún caso ha de significar claudicación, despolitización, demonización, marginación, soborno, o, especialmente, humillación. Por el contrario, ha de ser un proceso de dignificación de las personas que intervienen en él, pues han dejado las armas de manera voluntaria y como resultado de una negociación, un acuerdo o un cese de hostilidades (Fisas, V., 2011).

De acuerdo con lo anterior, la situación del reintegrado debe ser (aunque tampoco es asegurado) muy distinta a la de un desmovilizado. La transición es mucho más fácil, la oferta laboral debería ser más extensa y la posibilidad de seguir capacitándose debe ser aún mayor teniendo en cuenta que viene de contexto y unas condiciones sociales distintas e inferiores que las de un ciudadano promedio. El desmovilizado sabe convivir en sociedad, tiene un oficio o una profesión, es un ciudadano normal y debería ser tratado como tal, y sin embargo, es aquí donde más falla el proceso.

El sector privado suele ser odioso al momento de contratar gente reintegrada (y por lo tanto, lo es el doble con personas desmovilizadas) en sus empresas. El miedo, aunque psicológico en la mayoría de casos y problemas de estigma, hacen que el empleador no sea objetivo al momento de contratar, y cree que, por el pasado del desmovilizado, este es incapaz de realizar labores pertinentes a lo que es atención al cliente y servicios directos con el público, tal como lo afirma Roldan, L. (2013):

[...] los sectores que no tienen tendencia a vincular dentro de su nómina personas desmovilizadas argumentan principalmente que su condición de desmovilizados no es compatible con la naturaleza del servicio prestado, inseguridad de sus clientes y de la empresa, que no son un personal profesionalmente calificado, la confidencialidad de la información que manejan, la falta de confianza y el miedo e intranquilidad que les generan, los antecedentes de estas personas y porque prefieren elegir entre otras opciones (p.115).

Es aquí cuando entra en juego la dignidad del reintegrado. Debemos recordar varios puntos que vemos vulnerados a lo largo del proceso: primero, la suscripción del Acuerdo de Paz, que implican explícitamente que no hay vencedores; dos fuerzas se vieron en la necesidad de llegar a acuerdos, debido a que ni uno ni el otro logro derrotar al otro. Este es el primer principio que se viola en este proceso de reintegración. El reintegrado es visto ante el sector empresarial como un criminal, un enemigo derrotado acostumbrado a solo un tipo de actividad: el terrorismo.

Segundo, la falta de presencia del Estado al momento de crear políticas públicas para la vinculación de esta población al sector privado. Pueden existir exenciones tributarias (muy pocas pero las hay); sin embargo, el empresario prefiere pagarlas a tener que contar con desmovilizados en sus nóminas. Gracias a esto y al estigma manejado por el sector empresarial, no solo se ve afectada la dignidad humana del reintegrado, el cual se siente cosificado, definido y sin posibilidad de demostrar su esencia ante las actividades del diario vivir, y adicional al esto, se violan derechos constitucionales como lo son el derecho al trabajo y el principio de doble incriminación, el cual prohíbe que un sujeto sea castigado dos veces por el mismo delito; y sin embargo, el desmovilizado es por siempre castigado por sus delitos, sin importar que los saldó con el Estado y con él mismo.

Si bien, el postulado de dignidad humana, consiste en vivir bien, como se quiera, y sin humillaciones, algo se está haciendo mal. El desmovilizado siempre se ve afectado por su pasado, por su condición de desmovilizado, que bien debería ser transitorio, y no de por vida. La esencia de esa persona, de ese desmovilizado, se ve y verá opacada por aquel pasado, que ante el mundo empresarial lo define, y esto lesiona su dignidad y derechos como lo son del trabajo y la igualdad.

Si vamos por el argumento en el cual la constitución es norma de normas, en el preámbulo de la Carta Magna encontramos contemplado que el Estado Social de Derecho se encuentra fundado en los siguientes pilares: dignidad humana, trabajo y solidaridad. Estos postulados se ven vulnerados en el presente contexto: la dignidad, al momento de cosificar al reintegrado, el trabajo, al no darle la posibilidad al reintegrado de tener posibilidades como un ciudadano común, y la solidaridad, la cual el sector privado incumple al evitar este tipo de población en su nómina. Sin embargo, esto puede corregirse implementando diferentes políticas públicas que estén entrelazadas con estos postulados como con los acuerdos que se den 
entre el Estado y los grupos desmovilizados. ¿Y si en vez de capacitar al reintegrado como empleado, se capacita como empleador? Al generar empresa, por ejemplo, no solo se está cumpliendo con la garantía de ese reintegrado, sino que se genera empleo, lo cua no solo beneficiaría la población desmovilizada, sino la población en general, dando cumplimiento a lo enunciado en el Preámbulo.

\section{CONCLUSIONES}

En este orden de ideas, el concepto de igualdad tiene que dejar de ser el de una igualdad plana y genérica. La larga persistencia de la concepción de igualdad como "hecho", está en el origen de la configuración de la igualdad como asimilación de diferencias. Se debe tener en cuenta las diferencias que tienen estas minorías en relación con la población general, y hasta las diferencias que se encuentran internamente entre nuestra división del desmovilizado y reintegrado. Hobbes solía decir que todos los hombres son iguales porque todos mueren, y además porque se encuentran todos en la misma situación de hacerse daños unos a otros.

[... Pero existe también un cuarto modelo de configuración jurídica de las diferencias, el de la igual valoración jurídica de las diferencias, basado en el principio normativo de igualdad en los derechos fundamentales (políticos, civiles, de libertad y sociales) y al mismo tiempo en un sistema de garantías capaces de asegurar su efectividad (Ferrajoli, L., 1999).

En un país propenso a la desigualdad, debemos recurrir a alternativas que nos ayude a cumplir a cabalidad la reintegración exitosa estas minorías. Propender al pleno cumplimiento de sus derechos y garantías es la única forma de asegurar una verdadera terminación del conflicto.

El alto índice de deserción vivido en la desmovilización de las AUC es un indicador de alarma; un fenómeno a estudiar para asegurar que no se vuelva a repetir. Un grupo armado de medianamente unos 40.000 militantes, cuya población reintegrada no alcanza a ser el $10 \%$. Una derrota a las políticas públicas, un llamado a reorganizar y estructurar el camino a la reintegración.

La falta de vinculación laboral en el sector privado y las ofertas cuantiosas de grupos delictivos, son dos de las razones por las cuales la población en cuestión no termina el proceso, deserta de él, o muchas veces ni se involucra en ello; sin contar el largo proceso que es la reintegración: un camino de ocho años.

Los procesos de paz requieren varios cambios de la sociedad; es necesaria una convergencia total para que ello pueda ser totalmente exitosos.
La desmovilización, la reintegración y posterior convivencia en sociedad son etapas que necesitan de solidas políticas, que logren llevar nuevamente a estas minorías a una verdadera igualdad con los demás ciudadanos

Como ejemplo de lo anterior, en el Departamento de Caldas, solo un $16.3 \%$ de los reintegrados registrados en el departamento están vinculados formalmente al sector empresarial. Es decir que de 524 excombatientes registrados ante el departamento, solo 85 de ellos se encuentran en la formalidad (Aguirre, R., 2016). Junto con estas cifras y las cifras ya reportadas anteriormente de las AUC, está más que comprobado que los desmovilizados no tienen un verdadero umbral de igualdad. Si afirmamos entonces, como dice Bobbio, N., que la igualdad es la condición hacia la justica, con estos procesos no estamos creando sino más injusticias, más desigualdades y nuevos conflictos.

[...] la igualdad se debe tener como un tipo de relación entre los entes que forman parte de categoría abstracta de la humanidad; un fin para los componentes singulares de una totalidad. El único nexo social y políticamente relevante entre libertad e igualdad se confronta donde la libertad se considera como aquello en lo que los miembros de una comunidad son o deben ser iguales. Igualmente, libres, iguales en libertad" (Bobbio, N., 1993).

En un país propenso a la desigualdad, debemos recurrir a alternativas que nos ayude a cumplir a cabalidad la reintegración exitosa estas minorías. Propender al pleno cumplimiento de sus derechos y garantías es la única forma de asegurar una verdadera terminación del conflicto.

El alto índice de deserción vivido en la desmovilización de las AUC es un indicador de alarma; un fenómeno a estudiar para asegurar que no se vuelva a repetir. Un grupo armado de medianamente unos 40.000 militantes, cuya población reintegrada no alcanza a ser el $10 \%$. Una derrota a las políticas públicas, un llamado a reorganizar y estructurar el camino a la reintegración.

La falta de vinculación laboral en el sector privado y las ofertas cuantiosas de grupos delictivos, son dos de las razones por las cuales la población en cuestión no termina el proceso, deserta de él, o muchas veces ni se involucra en ello; sin contar el largo proceso que es la reintegración: un camino de ocho años.

Los procesos de paz requieren varios cambios de la sociedad; es necesaria una convergencia total para que ello pueda ser totalmente exitosos. La desmovilización, la reintegración y posterior convivencia en sociedad son etapas que necesitan de solidas políticas, que logren llevar nuevamente a estas minorías a una verdadera igualdad con los demás 
ciudadanos.

Como ejemplo de lo anterior, en el Departamento de Caldas, solo un $16.3 \%$ de los reintegrados registrados en el departamento están vinculados formalmente al sector empresarial. Es decir que de 524 excombatientes registrados ante el departamento, solo 85 de ellos se encuentran en la formalidad (Aguirre, R., 2016). Junto con estas cifras y las cifras ya reportadas anteriormente de las AUC, está más que comprobado que los desmovilizados no tienen un verdadero umbral de igualdad. Si afirmamos entonces, como dice Bobbio, N., que la igualdad es la condición hacia la justica, con estos procesos no estamos creando sino más injusticias, más desigualdades y nuevos conflictos.

[... la igualdad se debe tener como un tipo de relación entre los entes que forman parte de categoría abstracta de la humanidad; un fin para los componentes singulares de una totalidad. El único nexo social y políticamente relevante entre libertad e igualdad se confronta donde la libertad se considera como aquello en lo que los miembros de una comunidad son o deben ser iguales. Igualmente, libres, iguales en libertad" (Bobbio, N., 1993).

Podemos concluir entonces, que Colombia, es uno de los países de América Latina que más tiempo llevaba en conflicto; 50 largos años en constantes enfrentamientos con las FARC - EP y adyacente a este, conflictos con el ELN, EPL y AUC. Adicional a esto, Colombia es el segundo país más desigual en Latinoamérica. Siguiendo este orden de ideas, viendo este panorama general, no podemos evitar preocuparnos ante las adversidades que padecen minorías como estas.

Es aquí donde comenzamos a encontrar variables distintas a los de la población general; caracterizaciones y configuraciones sociales discriminadores, que comienzan generar los primeros vestigios de desigualdad.

Aquí afirmamos tal como lo dice Dworkin (s.f.) en su libro virtud soberana, y nos gusta complementar con el enunciado de Bobbio de su libro igualdad y libertad: "la igualdad es la virtud soberana de toda comunidad política, es el puente hacia una verdadera justicia".

$\mathrm{Al}$ encontrar una verdadera igualdad, un equilibrio en los procesos de estos desmovilizados, y los que están por venir, lograremos una verdadera justicia, en donde estos sujetos ya no tendrán que estar condicionados a un rotulo, ni tendrán que preocuparse sobre su pasado en las filas. La última etapa de este proceso de reintegración, debe ser en la que el reintegrado pasa a ser nuevamente un ciudadano igualitario, es ahí donde ya su proceso debe tener fin, y en donde ya debe disfrutar nuevamente de su vida en sociedad.

\section{REFERENCIAS BIBLIOGRÁFICAS}

I.

Aguirre, R. (2016). Encontrar empleo, reto para los desmovilizados. La patria. Recuperado de

https://www.lapatria.com/economia/en contrar-empleo-reto-para-los-desmoviliza dos-291225

II. $\quad$ Bobbio, N. (1993). Igualdad y Libertad. Barcelona: Ediciones Paidós.

III. Castañeda, L. A. (2012). Efectos de la violencia Paramilitar en la Comunidad Colombiana en los años 1990 al 2006. Bogotá: Universidad Militar Nueva Granada

IV. Cherta, J. M. (2011). Dimensiones de la igualdad en el pensamiento de A. de Tocqueville. Quaderns de filosofia $i$ ciencia, 125-136.

V. Dworkin, R. (s.f.). Virtud Soberana. Harvard.

VI. Ferrajoli, L. (1999). Derechos y garantías: la ley del más débil. Madrid: Trotta.

VII. Medina - Arbeláez, C. (2007). "La relación excombatientes - estado en el marco del proceso de desarme, desmovilización y reintegración en Colombia. Un debate permanente sobre participación política, ciudadanía y garantía de derechos". En: Francia www.institut-gouvernance.org

VIII. Motta, M. C. (2008). Reinserción y posconflicto: el "volver" de jóvenes ex combatientes a la vida civil. Cali: Universidad del Valle.

IX. Organización Internacional del Trabajo. (2002). Manual sobre oportunidades de formación y empleo para excombatientes. Recuperado de https://mafiadoc.com/manual-sobre-op ortunidades-de-formacion-y-empleo-para -_59da79a21723dd8b8f63983b.html

X. Rettberg, A. (2012). Construcción de paz en Colombia. Bogotá, Colombia: Ediciones Uniandes.

XI. Ribotta, S. (2010). Sobre la propuesta de igualdad en Norberto Bobbio. Huri-age.

\section{REFERENCIAS COMPLEMENTARIAS}

XII. Abitbol, P. (2005). El concepto de diseño institucional. Bogotá: Universidad Nacional de Colombia.

XIII. Anderson, M. B. (1999). Do no harm: how aid can support peace - or war.

XIV. Árevalo, W. (2014). El Espectador. Obtenido de El Espectador: https://www.elespectador.com/search/ noticias\%20politica\%20el\%C2\%ADreto \%C2\%ADde\%C2\%ADlegislar\%C2\%AD 
el\%C2\%ADposconflicto\%C2\%ADarticul \% 2 \%AD509148

XV. Calsamiglia, A. (1997). En defensa de Kelsen. Barcelona: Universitat Pompeu Fabra.

XVI. Calsamiglia, A. (s.f.). Sobre la teoría general de las normas.

XVII. Castellanos, L. R. (2013). la inclusión laboral de los desmovilizados del conflicto en Colombia: auténtico mecanismo emancipador de la violencia en Colombia. Univ. Estud. Bogotá, $107-122$.

XVIII. Donatto, C. M. (2012). Teoría de la legislación. México: Instituto de investigaciones jurídicas.

XIX. Garfias, R. P. (s.f.). Teoría general de la legislación.

XX. Hinestroza, M. P. (2009). Responsabilidad social empresarial e igualdad de oportunidades en el empleo: ¿altruismo o legalidad? Univ. Empresa, Bogotá, 244-251.

XXI. Instituto Catalan Internacional . (2014). Escenarios de posconflicto en Colombia. Barcelona.

XXII. La Patria. (2017). Encontrar empleo, reto para los desmovilizados. La Patria.

XXIII. Motta, M. C. (2008). Reinserción y posconflicto: el "volver" de jóvenes ex combatientes a la vida civil. Cali: Universidad del Valle.

XXIV. Pérez, T. H. (2011). Conflicto y posconflicto en Colombia: una mirada a la política de seguridad democrática. Separata Humanística, 129-150.

XXV. Robayo, M. L. (2017). Constitución e imperio de la ley en Colombia. Piélagus, 151-163.

XXVI. Tocqueville, A. d. (s.f.). La democracia en América.

XXVII. Velazquez, J. O. (2012). Reinserción social y función de la pena. UNAM.

XXVIII. Villa, H. V. (2005). La ley de justicia y y paz en Colombia a la luz del Derecho Internacional de los Derechos Humanos. Madrid. 